\title{
Multiscale gas-kinetic simulation for continuum and near-continuum flows
}

\author{
Kun $\mathrm{Xu}^{*}$ and Hongwei Liu \\ Department of Mathematics, The Hong Kong University of Science and Technology, Kowloon, Hong Kong, China
}

(Received 28 May 2006; revised manuscript received 20 November 2006; published 18 January 2007)

\begin{abstract}
It is well known that for increasingly rarefied flow fields, predictions from continuum formulations, such as the Navier-Stokes equations, lose accuracy. The inclusion of higher-order terms, such as Burnett or high-order moment equations, could improve the predictive capabilities of such continuum formulations, but there has been only limited success. Here, we present a multiscale model. On the macroscopic level, the flow variables are updated based on the mass, momentum, and energy conservation through the fluxes. On the other hand, the fluxes are constructed on the microscopic level based on the gas-kinetic equation, which is valid in both continuum and near-continuum flow regimes. Based on this model, the nonequilibrium shock structure, Poiseuille flow, nonlinear heat conduction problems, and unsteady Rayleigh problem will be studied. In the near-continuum flow regime, the current gas-kinetic simulation is more efficient than microscopic methods, such as the direction Boltzmann solver and direct-simulation Monte Carlo method. In the continuum flow limit, the current formulation will go back to the gas-kinetic Navier-Stokes flow solver automatically.
\end{abstract}

DOI: 10.1103/PhysRevE.75.016306

PACS number(s): 47.45. $-\mathrm{n}, 02.60 . \mathrm{Cb}, 02.70 . \mathrm{Bf}$

\section{INTRODUCTION}

The dynamical behavior of flows far from hydrodynamic equilibrium is an important subject of nonequilibrium thermodynamics, with many applications in science and engineering. The classification of various flow regimes is based on a dimensionless parameter-i.e., the Knudsen number, which is a measure of the degree of rarefaction of the medium. The Knudsen number $\mathrm{Kn}$ is defined as the ratio of the mean free path to a characteristic length scale of the system. In the continuum flow regime where $\mathrm{Kn}<0.001$, the NavierStokes equations with linear relations between stress and strain and Fourier's law for heat conduction are adequate to model the fluid behavior. For flows in the continuumtransition regime $(0.01<\mathrm{Kn}<1)$, the Navier-Stokes equations are known to be inadequate. This regime is important for many practical engineering problems, such as the simulation of microscale flows [1] and hypersonic flow around space vehicles in low Earth orbit [2]. Hence, there is a strong desire and requirement for accurate models which give reliable solutions with lower computational costs.

Currently, the direct-simulation Monte Carlo (DSMC) method is the most successful technique in the numerical prediction of low-density flows [3]. However, in the continuum-transition regime, especially for the low-speed flow, the DSMC method suffers from statistical noise in the bulk velocity because of the random molecular motion. When the bulk velocity is much slower than the thermal velocity, many independent samples are needed to eliminate the statistical scattering, as for microelectromechanical system (MEMS) simulations. In fact, for the air at room temperature, the standard deviation in the molecular speed is about $300 \mathrm{~m} / \mathrm{s}$, which would require approximately $9 \times 10^{6}$ independent samples in the DSMC method to reduce the scatter in the bulk velocity to $0.1 \mathrm{~m} / \mathrm{s}$. For MEMS gas flows that operate in the $\mathrm{mm} / \mathrm{s}$ range, the number of required

\footnotetext{
${ }^{*}$ Corresponding author. Electronic address: makxu@ust.hk
}

samples can grow into trillions. So the DSMC method is impractical in these cases. Also, the requirement of cell size being less than the particle mean free path in the DSMC method prevents it from being widely applied in the continuum flow regime, especially for flows with high Reynolds numbers.

Alternatively, many macroscopic continuum models have been intensively developed in the literature. These include the Navier-Stokes and Burnett equations from the ChapmanEnskog expansion, Grad's 13 moment equations, the regularized 13 equations, and many others [1]. In order to assess these continuum models, a few tests have been carried out [4]. They concluded that none of the models is commonly acceptable for rarefied flow simulations. Overall, the small length scales and slow bulk gas velocity combine to make the continuum solution inaccurate and the particle solution time consuming. Besides the DSMC and continuum models, many alternative approaches have also been proposed in recent years, such as the information preservation (IP) method $[5,6]$ and the lattice Boltzmann method (LBM) [7]. However, the IP method and LBM are mostly used for isothermal flows. Recently, by considering only the deviation from equilibrium, Baker and Hadjiconstantinou developed a variance reduction method for a Monte Carlo solution of the Boltzmann equation [8], where significant computational savings can be obtained.

The goal of this study is to present a multiscale simulation, which uses both the macroscopic (mass, momentum, and energy densities) and microscopic (gas distribution function and particle collision) descriptions. On the microscopic level, a flux is constructed based on a close solution of the gas-kinetic equation for an update of the flow variables on the macroscopic level.

\section{GAS-KINETIC EQUATION AND MULTISCALE NUMERICAL FORMULATION}

The Boltzmann equation expresses the behavior of a many-particle kinetic system in terms of the evolution equa- 
tion for a single-particle gas distribution function. The simplification of the Boltzmann equation given by the Bhatnagan-Gross-Krook (BGK) model is formulated as [9]

$$
\frac{\partial f}{\partial t}+\mathbf{u} \cdot \frac{\partial f}{\partial \mathbf{x}}=\frac{f^{e q}-f}{\tau},
$$

where $f$ is the number density of molecules at position $\mathbf{x}$ and particle velocity $\mathbf{u}=(u, v, w)$ at time $t$. The left-hand side of the above equation represents the free streaming of molecules in space, and the right-hand side denotes the collision term. If the distribution function $f$ is known, macroscopic variables, such as mass, momentum, energy, and stress, can be obtained by integration over the moments of molecular velocity. In the BGK model, the collision operator involves simple relaxation to a state of local equilibrium, the distribution function given by $f^{e q}$ with a characteristic time scale $\tau$. For a monotomic gas, the equilibrium state is given by a Maxwellian,

$$
f^{e q}=\rho\left(\frac{\lambda}{\pi}\right)^{3 / 2} e^{\left.-\lambda[\mathbf{u}-\mathbf{U})^{2}\right]},
$$

where $\rho$ is the density, $\mathbf{U}$ is the macroscopic fluid velocity, and $\lambda$ is equal to $m / 2 k T$. Here, $m$ is the molecular mass, $k$ is the Boltzmann constant, and $T$ is the temperature. The relation between mass $\rho$, momentum $\rho \mathbf{U}$, and energy densities $\rho E$ with the distribution function $f$ becomes

$$
\left(\begin{array}{c}
\rho \\
\rho \mathbf{U} \\
\rho E
\end{array}\right)=\int \psi_{\alpha} f d \mathbf{u},
$$

where $\psi_{\alpha}$ is the component of the vector of moments,

$$
\boldsymbol{\psi}=\left(1, \mathbf{u}, \frac{1}{2} \mathbf{u}^{2}\right)^{T}
$$

and the volume element in the phase space with $d \mathbf{u}=d u d v d w$ and $\mathbf{u}^{2}=u^{2}+v^{2}+w^{2}$. Since mass, momentum, and energy are conserved during particle collisions, $f$ and $f^{e q}$ satisfy the conservation constraint

$$
\int\left(f^{e q}-f\right) \psi_{\alpha} d \mathbf{u}=0
$$

at any point in space and time. The BGK model was originally proposed to describe the essential physics of molecular interactions with $\tau$ chosen as the molecular collision time. Although the BGK model appears to describe only weak departures from local equilibria, it has long been recognized that such an approximation works well beyond its theoretical limits as long as the relaxation time is known for the physical process. Based on the above BGK model, the Navier-Stokes equations can be derived with the Chapman-Enskog expansion truncated to first order,

$$
f=f^{e q}+\mathrm{Kn} f_{1}=f^{e q}-\tau\left(\partial f^{e q} / \partial t+\mathbf{u} \cdot \partial f^{e q} / \partial \mathbf{x}\right) .
$$

For the Burnett and super-Burnett solutions, the above expansion can be naturally extended [10], such as $f=f^{e q}+\mathrm{Kn} f_{1}+\mathrm{Kn}^{2} f_{2}+\mathrm{Kn}^{3} f_{3}+\cdots$.
Even though great progress has been made in the past two decades in the construction and analysis of the extended hydrodynamics system, the Burnett and super-Burnett equations, or regularization of the moment equations, the most successful method to accurately capture rarefied gas effect is still the DSMC method. The DSMC method is basically equivalent to solving the Boltzmann equation by simulating the free transport and collision steps in a particle transport process. The DSMC solution has the same solution as the full Boltzmann equation. However, based on the ChapmanEnskog expansion, only a limited number of truncated solutions are retained. Different from the Chapman-Enskog expansion, our present model is based on a closed solution of the kinetic equation. From the BGK model, we assume that it has the following closed solution:

$$
f=f^{e q}-\tau_{*}\left(\partial f^{e q} / \partial t+\mathbf{u} \cdot \partial f^{e q} / \partial \mathbf{x}\right),
$$

where $\tau_{*}$ is the parameter to be determined. The difference between the above solution and the first-order ChapmanEnskog expansion (4) is that a generalized collision time $\tau_{*}$ is introduced. Substituting the above equation into the BGK model (1), we can obtain the relation between the generalized particle collision time $\tau_{*}$ and the collision time $\tau$, which is well defined in the continuum flow regime,

$$
\tau_{*}=\frac{\tau\left(1-D \tau_{*}\right)}{1+\tau\left(D^{2} f^{e q} / D f^{e q}\right)},
$$

where $D=\partial / \partial t+\mathbf{u} \cdot \partial / \partial \mathbf{x}$. To the leading order, a simplified local collision time

$$
\tau_{*}=\frac{\tau}{1+\tau\left(D^{2} f^{e q} / D f^{e q}\right)}
$$

can be obtained. In the continuum flow limit, the modification term $\tau D^{2} f^{e q} / D f^{e q} \sim \mathrm{Kn}$ is expected to be small and $\tau_{*}$ reverts back to $\tau$, which is determined by $\tau=\mu / p$. The dynamic viscosity coefficient $\mu$ can be obtained experimentally or theoretically, such as Sutherland's law. In order to remove the dependence of the collision time $\tau_{*}$ on the individual molecular velocity, $D^{2} f^{e q} / D f^{e q}$ can be evaluated by taking a moment $\phi$, such as $\int \phi D^{2} f^{e q} d \mathbf{u} d \xi / \int \phi D f^{e q} d \mathbf{u} d \xi$. Since the stress and heat conduction terms result from the different moments of the gas distribution function, the value of $\tau_{*}$ in the viscosity term $\tau_{* p}$ and heat conduction coefficient $\tau_{*} p C_{p} / \mathrm{Pr}$ are obtained separately from different moments: $\phi_{1}=(\mathbf{u}-\mathbf{U})^{2}$ for the viscosity coefficient and $\phi_{2}=(u-U)\left[(\mathbf{u}-\mathbf{U})^{2}\right]$ for the heat conduction coefficient. A single moment was used in an early investigation of the argon shock structure calculation [11]. Since both $D^{2} f^{e q}$ and $D f^{e q}$ involve higher-order spatial and temporal derivatives of an equilibrium gas distribution function, a nonlinear limiter is imposed on the determination of $\tau_{*}$,

$$
\tau_{*}=\frac{\tau}{1+\max \left(-\alpha \mathrm{Kn}, \min \left(\tau\left(D^{2} f^{e q} / D f^{e q}\right), \alpha \mathrm{Kn}\right)\right)},
$$

where $\mathrm{Kn}$ is the Knudsen number and $\alpha$ takes the value 2 . The reason for introducing the above limiter is the following. First, since there are several terms involved in $D^{2} f^{e q} / D f^{e q}$, the mathematical evaluation of the ratio will be sensitive to 
the numerical error and large fluctuations will be generated, especially in the flow region close to equilibrium, where both first- and second-order derivatives tend to vanish. Also, the difference between $\tau_{*}$ and $\tau$ depends on the rarefaction of the flow. So, in order to avoid numerical singularity, a Knudsennumber-dependent limiter is used. With the above formulation, in the continuum flow limit-i.e., $\mathrm{Kn} \rightarrow 0$ - the modification to $\tau$ vanishes and the traditional definition $\tau$ is recovered.

The multiscale gas-kinetic scheme (GKS) for the nearcontinuum flow simulation is constructed based on the transfer between the macroscopic and microscopic flow descriptions. On the macroscopic level, the time evolution of the macroscopic variables can be achieved through a finitevolume formulation. For each control volume, such as $x \in\left[x_{j-1 / 2}, x_{j+1 / 2}\right]$ in the one-dimensional (1D) case, the update of conservative flow variables is

$$
\mathbf{W}_{j}^{n+1}=\mathbf{W}_{j}^{n}+\frac{1}{\Delta x} \int_{0}^{\Delta t}\left[\mathbf{F}_{j-1 / 2}(t)-\mathbf{F}_{j+1 / 2}(t)\right] d t,
$$

where $\mathbf{W}_{j}^{n}$ are the cell-averaged mass, momentum, and total energy and $\mathbf{F}_{j+1 / 2}$ are the corresponding fluxes at a cell interface, which is provided at microscopic level using the solution of the kinetic model,

$$
F=\int u \psi f d \mathbf{u} .
$$

Note that $\Delta t$ is the time step $\Delta t=t^{n+1}-t^{n}$. As an explicit numerical scheme, the time step of the GKS for high-Reynoldsnumber flows is determined by the Courant-Friedrichs-Lewy (CFL) condition-i.e., $\Delta t=\Delta t_{C F L} \equiv \eta \Delta x /\left(|U|_{\max }+c_{s}\right)$, where $0 \leq \eta \leq 1$ is the CFL number, $\Delta x$ is the minimum cell size, $|U|_{\max }$ is the maximum of the velocity, and $c_{s}=\sqrt{R T}$ is the sound speed. For low-Reynolds-number flows, the viscous term also influences the stability, and another criterion $\Delta t \leq(\Delta x)^{2} /\left(2^{D} \nu\right)$, with spatial dimension $D$, should be imposed on the time step. Therefore, a unified stability condition can be expressed as

$$
\Delta t \leq \frac{\eta \Delta x}{\left(|U|+c_{s}\right)\left(1+2 / \operatorname{Re}_{\Delta x}\right)},
$$

where $\operatorname{Re}_{\Delta x}=|U| \Delta x / \nu$ is the grid Reynolds number.

The microscopic model is mainly used to evaluate the gas distribution function at a cell interface. Equation (4) only presents a gas distribution function at an instant of time, such as the beginning of each time step $t^{n}=0$. In order to obtain a high-order accurate solution in the whole time step from $t^{n}$ to $t^{n+1}$, we need to evaluate a time-dependent gas distribution function, which can be constructed in the following [12]:

$$
f=f^{e q}-\tau_{*}\left(\partial f^{e q} / \partial t+u \partial f^{e q} / \partial x\right)+t \frac{\partial f^{e q}}{\partial t} .
$$

The relation between $\tau_{*}$ and $\tau$ is given in Eq. (7), where $\tau=\mu / p$ and $\mu$ is given by Sutherland's law. The timedependent part $t \partial f^{e q} / \partial t$ is used to account for the time evolution of the gas distribution function. In the 1D case, for a monotomic gas the equilibrium state $f^{e q}$ is
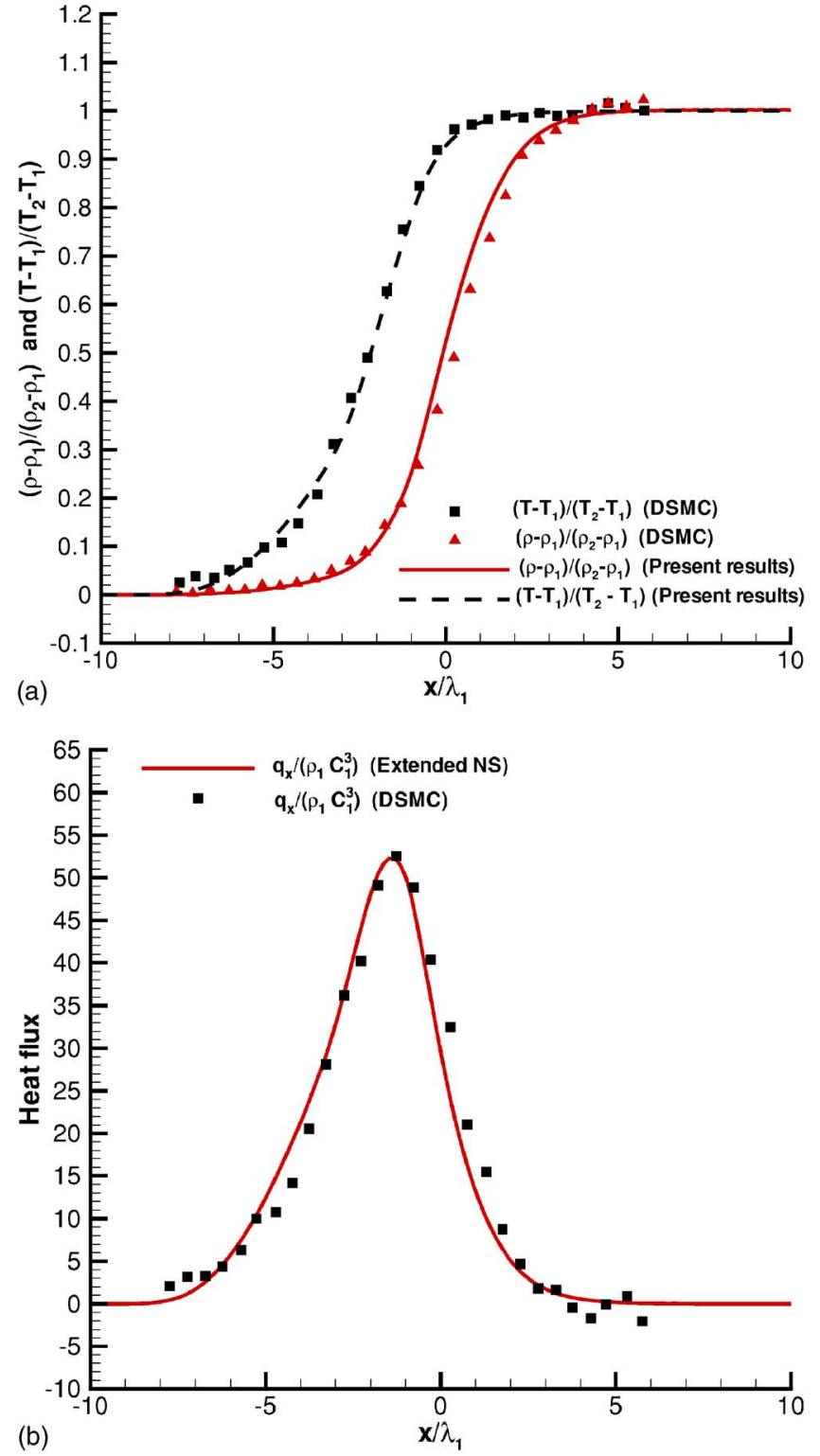

FIG. 1. (Color online) Temperature and density (a) and heat flux (b) distributions in a $M=8$ argon shock structure with $\mu \sim T^{0.68}$. DSMC solution [13] vs present multiscale model.

$$
f^{e q}=\rho\left(\frac{\lambda}{\pi}\right)^{3 / 2} \exp \left[-\lambda(\mathbf{u}-\mathbf{U})^{2}\right] .
$$

The expansion $\partial f^{e q} / \partial x$ can be expressed as

$$
\frac{\partial f^{e q}}{\partial x}=\left(a_{1}+a_{2} u+a_{3} \mathbf{u}^{2}\right) f^{e q}=a f^{e q} .
$$

Then, all the coefficients can be explicitly determined through the relation between the microscopic and macroscopic variables-i.e., $\mathbf{W}=\int \boldsymbol{\psi} f f^{e q} d \mathbf{u}$ and $\partial \mathbf{W} / \partial x=\int \psi a f^{e q} d \mathbf{u}$, where $\mathbf{W}=(\rho, \rho \mathbf{U}, \rho E)^{T}$ are the macroscopic mass, momentum, and energy densities. The temporal variation of $\partial f^{e q} / \partial t$ can be expanded similarly as a spatial expansion, and the corresponding coefficients can be obtained from the compatibility condition $\int\left(f-f^{e q}\right) \boldsymbol{\psi} d \mathbf{u}=0$-i.e., 

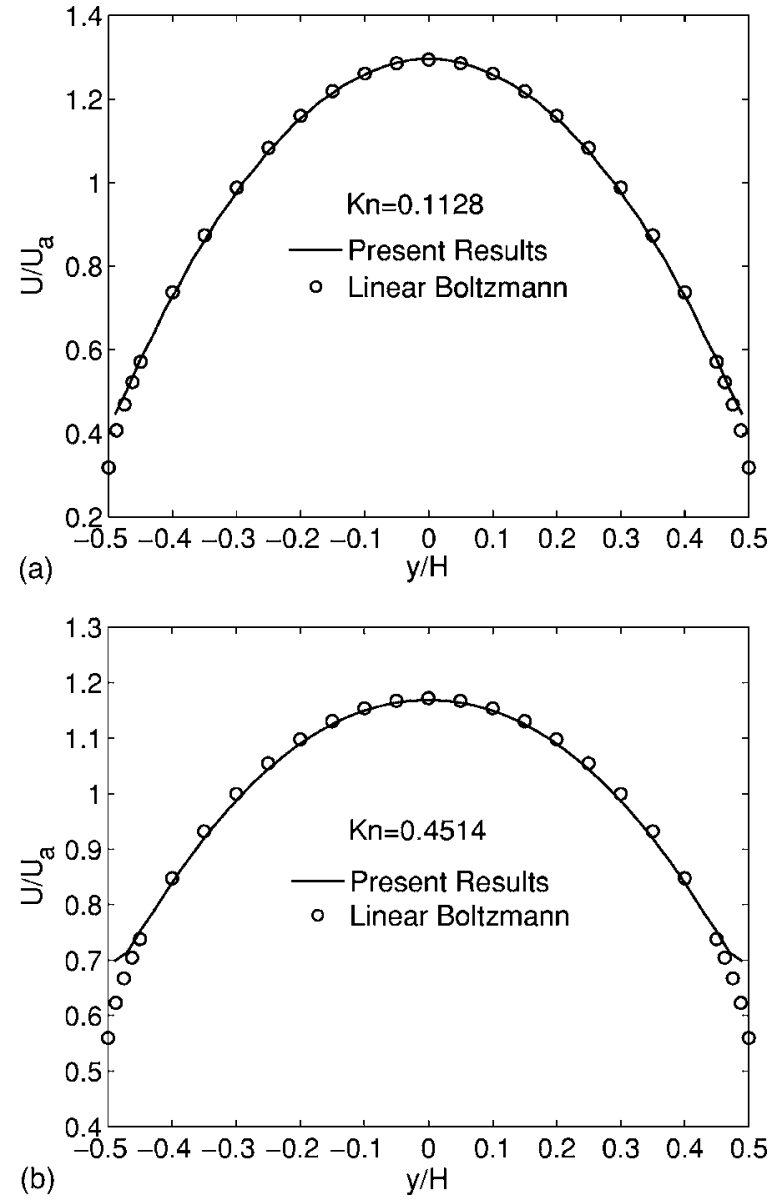

FIG. 2. Velocity distribution in the Poiseuille flow for HS molecules $\mu \sim T^{0.5}$, at two different Knudsen numebrs $\mathrm{Kn}=0.1128$ (a) and $\mathrm{Kn}=0.4514$ (b). Circles: solution of the Boltzmann equation [14]. Solid line: present multiscale model.

$$
\int \boldsymbol{\psi}\left(\partial f^{e q} / \partial t+u \partial f^{e q} / \partial x\right) d \mathbf{u}=0 .
$$

Therefore, on the microscopic level, a time-dependent gas distribution function can be uniquely constructed, which can be subsequently used in the evaluation of the corresponding fluxes.

In order to simulate the flow with any realistic Prandtl number, a modification of the heat flux in the energy transport, such as that used in [12], is also implemented in the present study.

\section{NUMERICAL EXPERIMENTS}

To test the correctness of the above multiscale model, we have studied a few cases for the near-continuum flow, which are the argon shock structure, Poiseuille flow for hard sphere (HS) molecules, nonlinear heat conduction problems for helium gas, and the unsteady Rayleigh problem.

For nonequilibrium flow, one of the simplest and most fundamental gas dynamic phenomena that can be used for model validation is the internal structure of a normal shock wave. There are mainly two reasons for this. First, the shock
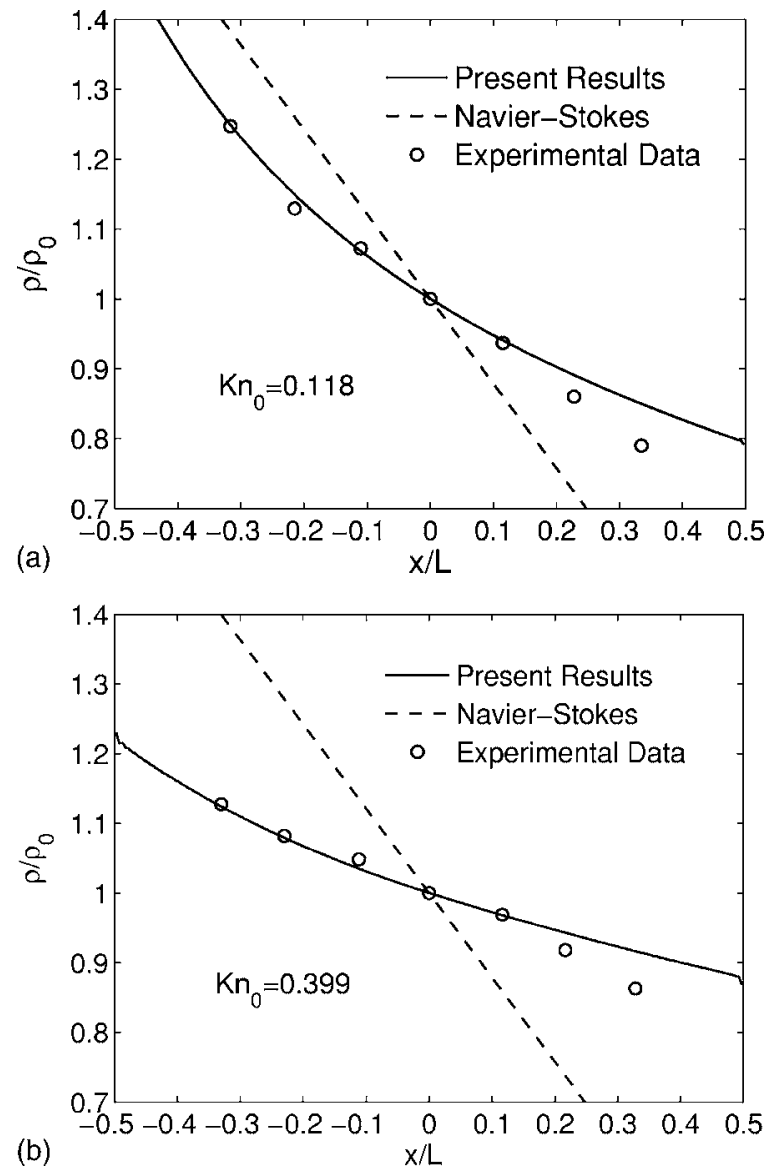

FIG. 3. Density ratio versus position for helium gas in a channel with temperatures $T_{H}=294 \mathrm{~K}$, and $T_{C}=79 \mathrm{~K}$ and accommodation coefficients $\alpha_{H}=\alpha_{C}=0.58$ at two different Knudsen numbers $\mathrm{Kn}=0.118$ (a) and $\mathrm{Kn}=0.399$ (b). Circles: experimental data [15]. Dashed line: Navier-Stokes solution. Solid line: present multiscale model.

wave represents a flow condition that is far from thermodynamic equilibrium, and second, the shock wave phenomenon is unique in that it allows one to separate the continuum differential equations of fluid motion from the boundary conditions. The boundary conditions for a shock wave are simply determined by the Rankine-Hugoniot relations. For the Mach-number-8 argon shock structure, Bird's [13] DSMC method using an inverse 11th-power repulsive potential $\mu \sim T^{0.68}$ gave good agreement with the experimental profile of argon gas. Figure 1 shows the solution of temperature, density, and heat flux from the current multiscale model compared with Bird's DSMC solutions. For this Mach-8 case, the shock thickness and the separation distance between the density and temperature profiles by the current model compare well with DSMC solution.

The Poiseuille flow is also a fundamental flow problem of rarefied gas dynamics, which is closely related to the flow in microchannel devices. An accurate analysis of this problem has been carried out by many authors [1]. One of the benchmark results is the solution of the Boltzmann equation, which has been obtained by Ohwada et al. [14] for HS molecules. For HS molecules, we have $\mu \sim \sqrt{T}$ to determine the dynamical viscosity coefficient. The boundary condition for 

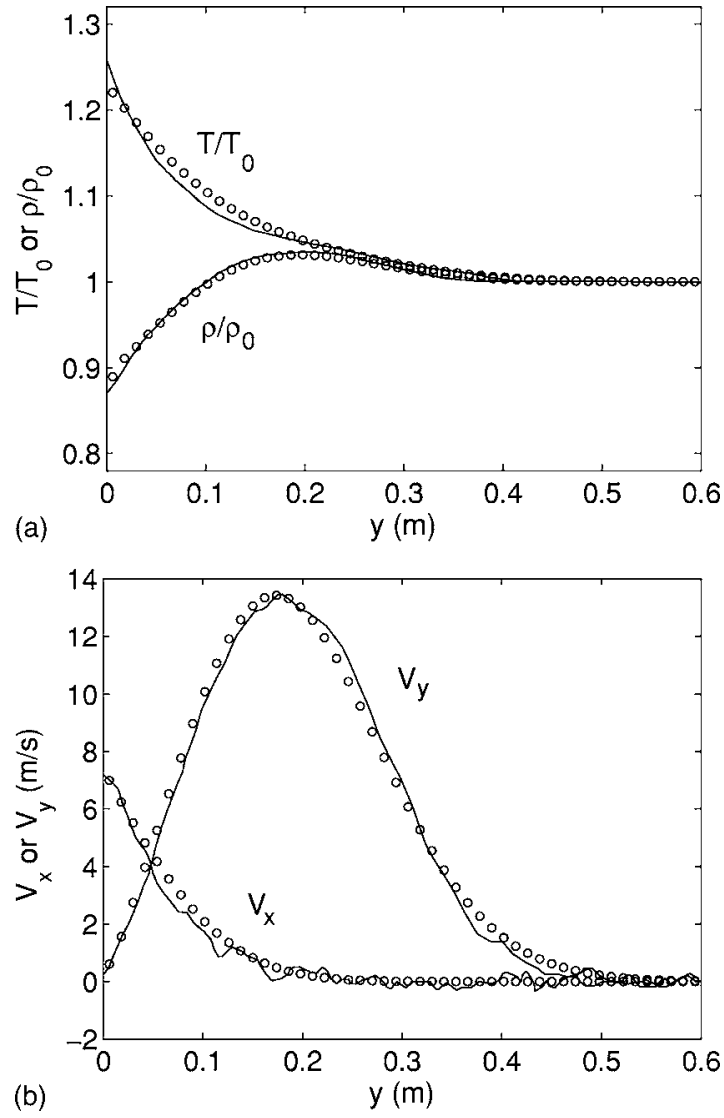

FIG. 4. Unsteady Rayleigh problem at time $t=10 \tau$. Solid lines: DSMC solutions [6]. Circles: present model.

the Poiseuille flow here is the Maxwell's diffusive boundary condition with accommodation coefficient 1 . The velocity distributions for both the Boltzmann equation and the current multiscale model are shown in Fig. 2 at two different Knudsen numbers. In the continuum flow regime-i.e., $\mathrm{Kn}=0.001$ - the current model automatically gives accurate Navier-Stokes solutions.

The next case is about the nonlinear heat transfer in the near-continuum flow regime. The experimental apparatus used in [15] consisted of two parallel flat plates made of aluminum, one cooled by liquid nitrogen and the other heated by electric heaters. The temperatures of the hot and cold plates were 294 and $79 \mathrm{~K}$, respectively. Helium gas was used as the test gas in the experiments. The Knudsen number is defined as the ratio of the mean free path at the center plane to the distance between the plates. For the boundary condition, the thermal accommodation coefficients measured in the free molecule regime in [16] were used with the assumption that their values remained constant over the entire range $(\alpha=0.58)$. The density distributions obtained from the current model and the continuum Navier-Stokes solution are compared with the experimental data in Fig. 3.

The last test is the Rayleigh problem. The Rayleigh flow is an unsteady flow in which a plate below a gas at rest suddenly acquires a constant parallel velocity and a constant temperature. In the current calculation [6], the argon gas is at rest at $t=0$ with a temperature of $273 \mathrm{~K}$. When $t>0$, the
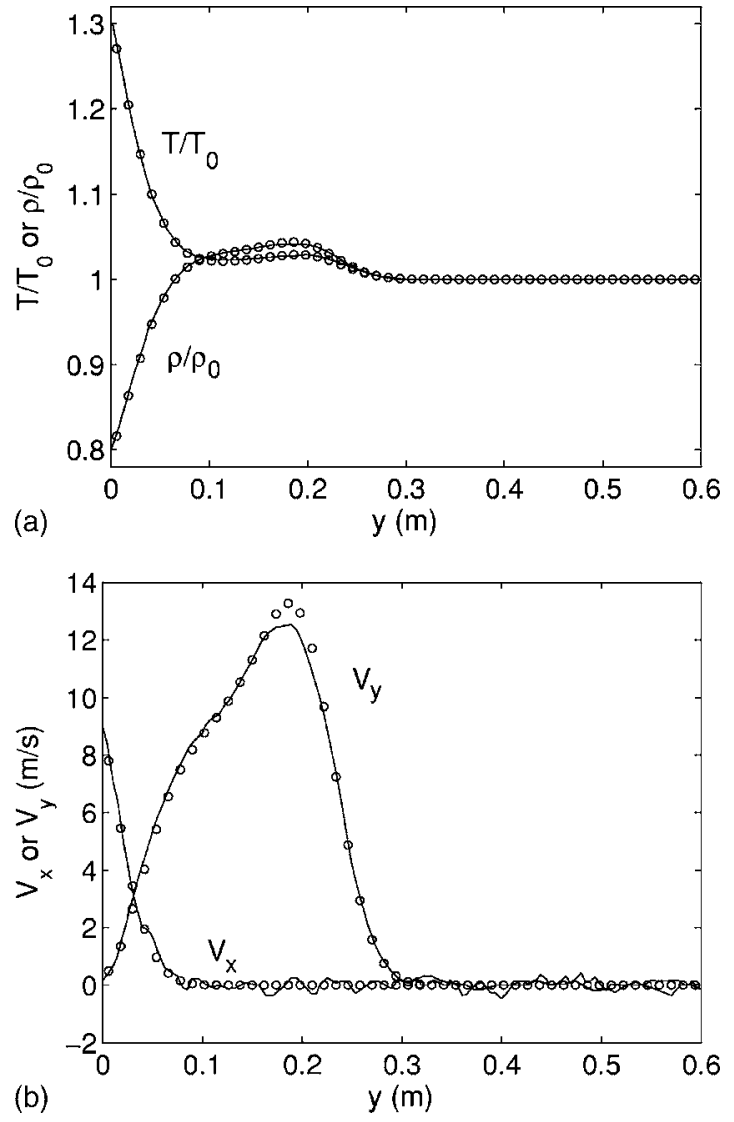

FIG. 5. Unsteady Rayleigh problem at time $t=100 \tau$. Solid lines: DSMC solutions [6]. Circles: present model.

plate obtains a constant velocity $10 \mathrm{~m} / \mathrm{s}$ and a constant temperature $373 \mathrm{~K}$. Figures 4 and 5 show the simulated solution for density, temperature, and velocities at two times of $t=10 \tau$ and $100 \tau$, where the agreement between the present model and the DSMC results is good at both times.

\section{CONCLUSION}

The multiscale method presented in this paper is a natural extension of the gas-kinetic method for the Navier-Stokes solutions to the near-continuum flow regime. The distinguishable point of the current kinetic model is that a closed solution of the BGK equation is used in the evaluation of a generalized particle collision time, which subsequently adjusts the values of the dissipative coefficients, such as the viscosity and heat conduction coefficients, in the nearcontinuum flow regime. This model has been successfully applied to a few nonequilibrium flow problems, such as the shock structure, Poiseuille flow, and unsteady Rayleigh problems. The merit of a multiscale method is due to the coupling between macroscopic and microscopic flow descriptions. The conservative flow variables are updated on the macroscopic level through the fluxes, which are evaluated on the microscopic level, where the flow physics can be much easily implemented. For the near-continuum flow, the DSMC 
method is very expensive and the continuum formulations are inaccurate. The current gas-kinetic method provides such a method, which has similar efficiency as the Navier-Stokes flow solvers, but with the flexibility in the modeling of the nonequilibrium effect on the particle distribution level. Furthermore, the current method can be naturally extended to include multiple translational, rotational, and vibrational temperatures, where the macroscopic governing equations are not well defined.

\section{ACKNOWLEDGMENT}

This research was supported by Hong Kong Research Grant Council, Grants Nos. HKUST6102/04E and 621005.

051703 (2005).

[9] P. L. Bhatnagar, E. P. Gross, and M. Krook, Phys. Rev. 94, 511 (1954).

[10] T. Ohwada and K. Xu, J. Comput. Phys. 201, 315 (2004).

[11] K. Xu, Phys. Fluids 14, L17 (2002).

[12] K. Xu, J. Comput. Phys. 171, 289 (2001).

[13] G. Bird, Phys. Fluids 13, 1172 (1970).

[14] T. Ohwada, Y. Sone, and K. Aoki, Phys. Fluids A 1, 2042 (1989).

[15] D. J. Alofs, R. C. Flagan, and G. S. Springer, Phys. Fluids 14, 529 (1971).

[7] F. Toschi and S. Succi, Europhys. Lett. 69, 549 (2005).

[8] L. L. Baker and N. G. Hadjiconstantinou, Phys. Fluids 17,

[16] W. P. Teagan and G. S. Springer, Phys. Fluids 11, 497 (1968). 